\title{
ALMA and NOEMA constraints on synchrotron nebular emission from embryonic superluminous supernova remnants and radio-gamma-ray connection
}

Kohta Murase ${ }^{\circledR}, 1,2,3,4 \star$ Conor M. B. Omand, ${ }^{5}$ Deanne L. Coppejans, ${ }^{6}$ Hiroshi Nagai, ${ }^{7,8}$ Geoffrey C. Bower, ${ }^{9,10}$ Ryan Chornock, ${ }^{11}$ Derek B. Fox, ${ }^{2,3}$ Kazumi Kashiyama, ${ }^{5,12}$ Casey Law, ${ }^{13}$ Raffaella Margutti ${ }^{12}$ and Peter Mészáros ${ }^{1,2,3}$

${ }^{1}$ Department of Physics, The Pennsylvania State University, University Park, PA 16802, USA

${ }^{2}$ Department of Astronomy and Astrophysics, The Pennsylvania State University, University Park, PA 16802, USA

${ }^{3}$ Center for Mulitmessenger Astrophysics, Institute for Gravitation and the Cosmos, The Pennsylvania State University, University Park, PA 16802, USA

${ }^{4}$ Center for Gravitational Physics, Yukawa Institute for Theoretical Physics, Kyoto University, Sakyo-ku, Kyoto 606-8502, Japan

${ }^{5}$ Department of Physics, Gradaute School of Science, University of Tokyo, Bunkyo-ku, Tokyo 113-0033, Japan

${ }^{6}$ Center for Interdisciplinary Exploration and Research in Astrophysics (CIERA); Department of Physics and Astronomy, Northwestern University, Evanston, IL 60208, USA

${ }^{7}$ National Astronomical Observatory of Japan, Mitaka, Tokyo 181-8588, Japan

${ }^{8}$ The Graduate University for Advanced Studies, SOKENDAI, Mitaka, Tokyo 181-8588, Japan

${ }^{9}$ Academia Sinica Institute of Astronomy and Astrophysics, Hilo, HI 96720, USA

${ }^{10}$ Department of Physics and Astronomy, University of Hawai'i at Mãnoa, Honolulu, HI 96822, USA

${ }^{11}$ Astrophysical Institute; Department of Physics and Astronomy, Ohio University, Athens, OH 45701, USA

${ }^{12}$ Research Center for the Early Universe; Department of Physics, Graduate School of Science, University of Tokyo, Bunkyo-ku, Tokyo 113-0033, Japan

${ }^{13}$ Cahill Center for Astronomy and Astrophysics, California Institute of Technology, Pasadena, CA 91125, USA

Accepted 2021 August 24. Received 2021 August 21; in original form 2021 May 12

\begin{abstract}
Fast-rotating pulsars and magnetars have been suggested as the central engines of superluminous supernovae (SLSNe) and fast radio bursts, and this scenario naturally predicts non-thermal synchrotron emission from their nascent pulsar wind nebulae (PWNe). We report results of high-frequency radio observations with ALMA and NOEMA for three SLSNe (SN 2015bn, SN 2016ard, and SN 2017egm), and present a detailed theoretical model to calculate non-thermal emission from PWNe with an age of $\sim 1-3 \mathrm{yr}$. We find that the ALMA data disfavours a PWN model motivated by the Crab nebula for SN 2015bn and SN 2017 egm, and argue that this tension can be resolved if the nebular magnetization is very high or very low. Such models can be tested by future $\mathrm{MeV}-\mathrm{GeV}$ gamma-ray telescopes such as AMEGO.
\end{abstract}

Key words: radiation mechanisms: non-thermal - stars: magnetars - pulsars: general - fast radio bursts - transients: supernova radio continuum: transients.

\section{INTRODUCTION}

Recent observations have revealed the diversity among different types of core-collapse supernovae ( $\mathrm{SNe}$ ) and compact binary mergers. Superluminous supernovae (SLSNe) are among the most luminous explosive phenomena, and their optical emission is likely to be powered by the central engine and/or interactions between the SN ejecta and circumstellar material (Moriya, Sorokina \& Chevalier 2018; Gal-Yam 2019; Chen 2021, for reviews). In particular, the most popular explanation for Type I SLSNe (SLSNe-I) that are not accompanied by hydrogen signatures is the 'pulsar/magnetar-driven' scenario, in which optical photons are radiated via thermalization of the rotation energy injected through pulsar winds (Chatzopoulos et al. 2013; Inserra et al. 2013; Nicholl et al. 2013). In general, the pulsar/magnetar engine is of interest in light of the diversity

${ }^{\star}$ E-mail: murase@psu.edu of transient phenomena (e.g. Thompson, Chang \& Quataert 2004; Zhang 2014; Greiner et al. 2015; Metzger et al. 2015; Kashiyama et al. 2016; Margalit et al. 2018), since it potentially gives a unified view of SLSNe, stripped-envelope SNe, long gamma-ray bursts (GRBs), and even fast radio bursts (FRBs). It has also been invoked to explain some of the rapidly rising optical transients (Hotokezaka, Kashiyama \& Murase 2017).

It is known that pulsar wind nebulae (PWNe) such as the Crab nebula are efficient accelerators of electrons and positrons (leptons) and possibly also ions. Broad-band non-thermal emission from the nebulae has been observed in many Galactic PWNe, suggesting that a significant fraction of the wind magnetic energy is used for particle acceleration around the termination shock (see a review, Gaensler \& Slane 2006). At early times, efficient thermalization of non-thermal photons occurs, and the observed SN light curves can be explained by adjusting the magnetic field $B$, initial period $P_{i}$, and ejecta mass $M_{\text {ej }}$ (e.g. Kasen \& Bildsten 2010; Woosley 2010). However, there 


\section{pulsar/magnetar-driven supernova}

$\sim$ a few months after the explosion

\section{embryonic pulsar/magnetar wind nebula \\ $\sim$ a few years after the explosion}
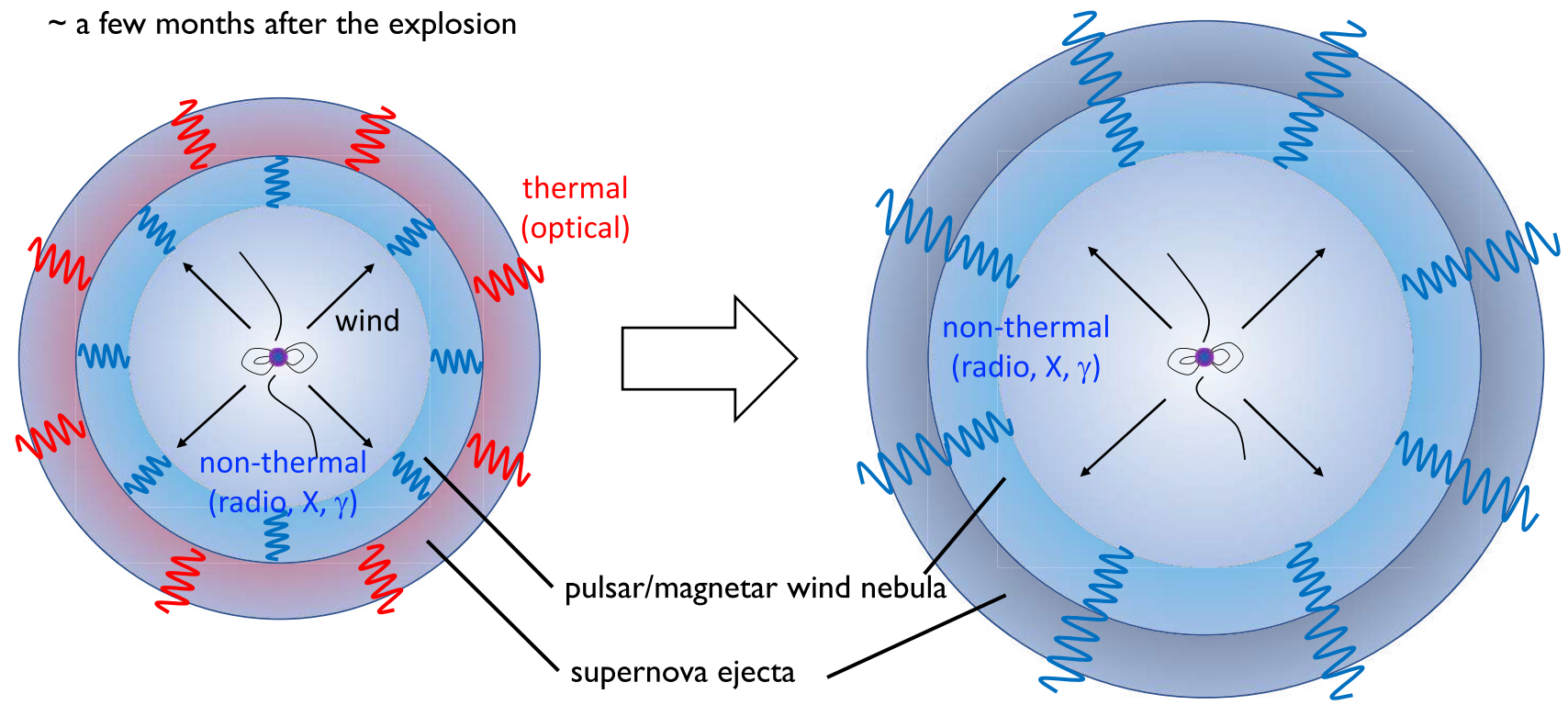

Figure 1. Schematic picture of non-thermal radiation from embryonic SN remnants powered by pulsar/magnetar winds. Radio and gamma-ray emission is observable when the system is optically thin to various processes.

is a large degeneracy in model parameters; and detecting highenergy signals is relevant for revealing not only the central engine but also particle acceleration at hidden environments (e.g. Murase, Mészáros \& Zhang 2009; Kotera, Phinney \& Olinto 2013; Murase et al. 2015).

Searches for non-thermal signatures from nascent magnetars have been further motivated by FRB studies. If FRBs originate from young neutron stars or white dwarfs, they would be expected to occur inside nebulae. Murase, Kashiyama \& Mészáros (2016) proposed quasisteady synchrotron emission as a probe of the FRB progenitors and their possible connection to pulsar/magnetar-driven $\mathrm{SNe}$ including SLSNe (see Fig. 1 for the schematic picture). Later, a persistent radio counterpart to FRB 121102 (Tendulkar et al. 2017) was reported (see reviews, Petroff, Hessels \& Lorimer 2019; Xiao, Wang \& Dai 2021 , for recent developments). On the other hand, X-ray and radio observations of SLSNe have yielded upper limits (Bhirombhakdi et al. 2018; Coppejans et al. 2018; Margutti et al. 2018; Law et al. 2019; Eftekhari et al. 2020), apart from SN 2017ens (Coppejans et al. 2021a), SN 2020tcw (Matthews et al. 2021; Coppejans et al. 2021b), and PTF 10hgi. The radio emission from PTF 10hgi may be explained by the nebular synchrotron emission (Eftekhari et al. 2019, 2020; Law et al. 2019). Nascent nebular emission from binary neutron star mergers (Murase et al. 2018; Yamasaki, Totani \& Kiuchi 2018; Margalit, Berger \& Metzger 2019) and accretion-induced collapse (Kashiyama \& Murase 2017; Margalit et al. 2019) has also been discussed.

This work presents new results of ALMA and NOEMA observations in the $90-230 \mathrm{GHz}$ bands, together with a numerical model to describe the early non-thermal nebular emission and its simple analytical prescription. We demonstrate the power of such high-frequency radio data, given that the detectability at the $\mathrm{GHz}$ band is often limited by strong absorption. We suggest models that can avoid existing multiwavelength constraints from radio to $\mathrm{X}$ ray bands, pointing out the importance of the connection to soft gamma-rays.

We assume cosmological parameters with $h=0.7, \Omega_{m}=0.3$, and $\Omega_{\Lambda}=0.7$. We also use notations as $Q_{x}=Q / 10^{x}$ in the CGS unit except $t_{\mathrm{yr}} \equiv(t / 1 \mathrm{yr})$ and $M_{\mathrm{ej}, 1}=M_{\mathrm{ej}} / 10 \mathrm{M}_{\odot}$.

\section{SYNCHROTRON EMISSION FROM EMBRYONIC SLSN REMNANTS}

\subsection{Overview}

A highly magnetized pulsar/magnetar may be left over as a compact remnant after the SN explosion. Either rotational or magnetic energy is extracted from it by a relativistic wind, forming an embryonic PWN and powering the $\mathrm{SN}$ emission in the optical band via dissipation and thermalization of the magnetic energy. The spin-down power is estimated by (Gruzinov 2005; Spitkovsky 2006)

$L_{\mathrm{sd}} \approx 7.2 \times 10^{41} \mathrm{erg} \mathrm{s}^{-1} B_{*, 13}^{2} P_{-2}^{-4}$,

where $B_{*}$ is the dipole magnetic field, $P(t)=P_{i}\left(1+t / t_{\mathrm{sd}}\right)^{1 / 2}$ is the spin period, and the neutron star radius is assumed to be $12 \mathrm{~km}$. The spindown time is $t_{\mathrm{sd}} \approx 0.12 \mathrm{yr} B_{*, 13}^{-2} P_{i,-3}^{2}$. An embryonic PWN is also expected to be a natural source of $\gamma$-rays and hard X-rays (photon energies $E_{\gamma}=h v \gtrsim 100 \mathrm{keV}$ range) with a long duration from months to years after the explosion (Murase et al. 2015; Kashiyama et al. 2016). In addition, $\sim 1-10 \mathrm{yr}$ after the explosion, quasi-steady synchrotron emission has been predicted to be detectable at the highfrequency radio band (Omand, Kashiyama \& Murase 2018).

PWNe aid the expansion of the SN ejecta; and the nebular size $R_{\mathrm{nb}}$ is typically smaller than the ejecta radius $R_{\mathrm{ej}}$ (e.g. Chevalier $\&$ Fransson 1992; Slane 2017). If the system is rotationally powered, one expects 
$R_{\mathrm{nb}} \approx R_{\mathrm{ej}} \approx \beta_{\mathrm{ej}} c t \approx \sqrt{2 \mathcal{E}_{\mathrm{rot}} / M_{\mathrm{ej}}} t \simeq 5.2 \times 10^{16} \mathrm{~cm} P_{i,-3}^{-1} M_{\mathrm{ej}, 1}^{-1 / 2} t_{\mathrm{yr}}$ for $t>t_{\mathrm{sd}}$. Then, the magnetic field accumulated in the nebula is estimated to be $B_{\mathrm{nb}} \approx\left(6 \epsilon_{B} \mathcal{E}_{\mathrm{rot}} / R_{\mathrm{nb}}^{3}\right)^{1 / 2} \simeq 1.9 \mathrm{G} \epsilon_{B,-2.5}^{1 / 2} P_{i,-3}^{1 / 2} M_{\mathrm{ej}, 1}^{3 / 4} t_{\mathrm{yr}}^{-3 / 2}$, where $\epsilon_{B} \sim 0.003$ is the energy fraction carried by nebular magnetic fields, as suggested for the Crab nebula (e.g. Kennel \& Coroniti 1984; Tanaka \& Takahara 2010). Note that this value is highly uncertain in embryonic PWNe, and that we consider different magnetization cases in our treatment below.

The typical Lorentz factor of radio-emitting leptons is

$\gamma(v) \simeq 110 \epsilon_{B,-2.5}^{-1 / 4} P_{i,-3}^{-1 / 4} M_{\mathrm{ej}, 1}^{-3 / 8} t_{\mathrm{yr}}^{3 / 4} \nu_{11}^{1 / 2}$.

The cooling Lorentz factor is given by $\gamma_{c}=6 \pi m_{e} c /\left(\sigma_{T} B_{\mathrm{nb}}^{2} t\right) \simeq$ $6.8 \epsilon_{B,-2.5}^{-1} P_{i,-3}^{-1} M_{\mathrm{ej}, 1}^{-3 / 2} t_{\mathrm{yr}}^{2}$. Thus, the synchrotron spectrum of embryonic PWNe is in the fast cooling regime, implying that the spectrum is softer than that of the Crab nebula with $\beta \sim 1.3$ in the radio band. Here, the photon index introduced by $F_{v} \propto v^{1-\beta}$ leads to $\beta=\max \left[3 / 2,\left(2+q_{1}\right) / 2\right]$, where $q_{1}<2$ is the low-energy spectral index of non-thermal leptons. The multiwavelength modelling of young Galactic PWNe implies that the leptons are accelerated to $\gamma_{b} \in\left[3 \times 10^{4}, 10^{7}\right]$ (Tanaka \& Takahara 2013). The characteristic synchrotron frequency, at which $v F_{v}$ peaks, is

$v_{b} \approx \frac{3}{4 \pi} \gamma_{b}^{2} \frac{e B_{\mathrm{nb}}}{m_{e} c} \simeq 3.2 \times 10^{18} \mathrm{~Hz} \gamma_{b, 5.8}^{2} \epsilon_{B,-2.5}^{1 / 2} P_{i,-3}^{1 / 2} M_{\mathrm{ej}, 1}^{3 / 4} t_{\mathrm{yr}}^{-3 / 2}$,

given that this is lower than the maximum synchrotron frequency, $v_{M} \sim 3.8 \times 10^{22} \mathrm{~Hz}$. Taking the reference frequency $v_{0}$ as the peak of $v F_{v}$ (i.e. $\left.v_{0}=\max \left[v_{\mathrm{b}}, v_{\mathrm{M}}\right]\right), F_{v}$ in the fast cooling limit is

$F_{\nu}=F_{\nu 0}\left(\frac{v}{v_{0}}\right)^{1-\beta} \approx \frac{\epsilon_{e} L_{\mathrm{sd}}}{8 \pi d^{2} v_{0} \mathcal{R}_{0}\left(1+Y_{\mathrm{IC}}\right)}\left(\frac{v}{v_{0}}\right)^{1-\beta}$,

where $\epsilon_{e} \sim 1$ is the energy fraction carried by the nonthermal leptons, $\mathcal{R}_{0}$ is a correction factor for the lepton normalization from the differential to bolometric powers, $Y_{\mathrm{IC}}$ is the Compton $\mathrm{Y}$ parameter, and $d$ is the distance to the source. In the case of $\beta=7 / 4$ (or $q_{1}=3 / 2$ ), we have $F_{v} \simeq$ $6.0 \mathrm{mJy} \quad \gamma_{b, 5.8}^{-1 / 2} \epsilon_{B,-2.5}^{-1 / 8} \nu_{11}^{-3 / 4} B_{*, 13}^{-2} P_{i,-3}^{-1 / 8} M_{\mathrm{ej}, 1}^{-3 / 16} t_{\mathrm{yr}}^{-13 / 8}(d / 0.5 \mathrm{Gpc})^{-2}$ $\left[\mathcal{R}_{0}\left(1+Y_{\mathrm{IC}}\right) / 4\right]^{-1}$, agreeing with numerical results (see below).

Radio emission is subject to various absorption processes. In particular, synchrotron (self-)absorption (SSA) and free-free absorption are relevant. The SSA frequency $v_{\mathrm{sa}}$ can be estimated by

$\pi \frac{R_{\mathrm{nb}}^{2}}{d^{2}} 2 k T_{\mathrm{sa}} \frac{v_{\mathrm{sa}}^{2}}{c^{2}}=F_{\nu 0}\left(\frac{v_{\mathrm{sa}}}{v_{0}}\right)^{1-\beta}$,

where

$T_{\mathrm{sa}}=\frac{1}{3 k} C\left(\frac{4 \pi m_{e} c v_{\mathrm{sa}}}{3 e B}\right)^{1 / 2} m_{e} c^{2}$

is the brightness temperature at $v_{\mathrm{sa}}$ and $C$ is an order-of-unity correction factor. We approximately have

$v_{\mathrm{sa}} \sim\left(\frac{3^{3 / 2} e^{1 / 2} B_{\mathrm{nb}}^{1 / 2} F_{v_{0}} v_{0}^{\beta-1} d^{2}}{4 \pi^{3 / 2} m_{e}^{3 / 2} c^{1 / 2} R_{\mathrm{nb}}^{2}}\right)^{\frac{2}{2 \beta+3}}$,

which leads to $v_{\mathrm{sa}} \sim 32 \mathrm{GHz} \epsilon_{B,-2.5}^{1 / 26} \gamma_{b, 5.8}^{-2 / 13} B_{*, 13}^{-8 / 13} P_{i,-3}^{17 / 26} M_{\mathrm{ej}, 1}^{19 / 52}$ $t_{\mathrm{yr}}^{-35 / 26}\left[\mathcal{R}_{0}\left(1+Y_{\mathrm{IC}}\right) / 4\right]^{-4 / 13}$ for $\beta=7 / 4$, agreeing with numerical results presented below. In reality, non-thermal emission escaping from the nebula is further degraded by free-free absorption in the SN ejecta, and the nebula typically becomes transparent at the $100 \mathrm{GHz}$ band 1-10 yr after the explosion (Murase et al. 2016; Margalit et al. 2018; Omand et al. 2018)

\subsection{Theoretical Model}

We here outline the theoretical model used for calculations of thermal and non-thermal spectra. Although the method used is similar to that of Omand et al. (2018) and Eftekhari et al. (2020), we review it so that we may provide details not presented in these works. Also, there are several improvements compared to Murase et al. (2016). In the pulsar/magnetar-powered SN scenario, a significant fraction of the spin-down energy needs to be deposited in the SN ejecta, and the evolution of the internal energy $\mathcal{E}_{\text {int }}$ is given by

$\frac{\mathrm{d} \mathcal{E}_{\text {int }}}{\mathrm{d} t}=f_{\mathrm{dep}, \mathrm{sd}} \epsilon_{e} L_{\mathrm{sd}}+f_{\mathrm{dep}, \mathrm{rd}} L_{\mathrm{rd}}-L_{\mathrm{sn}}-\frac{\mathcal{E}_{\mathrm{int}}}{R_{\mathrm{ej}}} \frac{\mathrm{d} R_{\mathrm{ej}}}{\mathrm{d} t}$,

where $L_{\mathrm{rd}}$ is the radioactive decay power, $f_{\mathrm{dep}, \mathrm{sd} / \mathrm{rd}}$ is the energy fraction deposited into thermal energy, $L_{\mathrm{sn}}$ is the $\mathrm{SN}$ luminosity, and the last term represents adiabatic losses. We estimate $f_{\text {dep,sd }}$ as in Kashiyama et al. (2016), but the treatment is improved by considering arbitrary $\gamma_{b}$ and $q_{1}<2$ rather than assuming $q_{1}=2$.

Dynamics of PWNe and SN ejecta can be calculated by solving equations of motion for the shocked shells (Ostriker \& Gunn 1971). Murase et al. (2016) studied radio and millimeter emission from nascent magnetars using analytical solutions for the PWN evolution. Instead, we estimate radii of the nebula and ejecta by solving the simplified equations (Metzger et al. 2014; Kashiyama et al. 2016)

$\begin{aligned} \frac{\mathrm{d} R_{\mathrm{nb}}}{\mathrm{d} t} & =V_{\mathrm{nb}}^{\prime}+\frac{R_{\mathrm{nb}}}{t}, \\ \frac{\mathrm{d} R_{\mathrm{ej}}}{\mathrm{d} t} & =V_{\mathrm{ej}},\end{aligned}$

where $V_{\mathrm{nb}}^{\prime}$ is the nebular velocity in the ejecta rest frame and $V_{\mathrm{ej}}$ is the ejecta velocity, respectively. For engine-powered SLSNe, the ejecta and nebula move together, i.e. $R_{\mathrm{ej}} \approx R_{\mathrm{nb}}$. The evolution of nebular magnetic fields is given by

$\frac{\mathrm{d} \mathcal{E}_{B}}{\mathrm{~d} t}=\epsilon_{B} L_{\mathrm{sd}}-c_{B} \frac{\mathcal{E}_{B}}{R_{\mathrm{nb}}} \frac{\mathrm{d} R_{\mathrm{nb}}}{\mathrm{d} t}$,

where $\mathcal{E}_{B}$ is the magnetic energy inside the nebula. The magnetic field is uncertain and the toroidal component may be accumulated in the nebula. In this work, we consider the limit $c_{B}=0$, as used in the modelling of Galactic PWNe (Tanaka \& Takahara 2010) and our past works (Murase et al. 2015; Omand et al. 2018).

Regarding thermal emission, the SN luminosity is calculated by

$L_{\mathrm{sn}}=\frac{\mathcal{E}_{\text {int }}}{t_{\mathrm{esc}}^{\mathrm{ej}}}$,

where the thermal photon escape time is

$t_{\mathrm{esc}}^{\mathrm{ej}}=\min \left[1, \tau_{T}^{\mathrm{ej}}\right] \frac{R_{\mathrm{ej}}}{c}$,

and $\tau_{T}^{\mathrm{ej}}$ is the Thomson optical depth for the SN ejecta. The ejecta temperature is set to the blackbody temperature (see Appendix C of Kashiyama et al. 2016). The SN thermal photons serve as targets for non-thermal photons, for which the late-time temperature is assumed to be no lower than $10^{4} \mathrm{~K}$.

We calculate intrinsic non-thermal emission from the nebula by solving the following kinetic equations

$\dot{n}_{E_{e}}^{e}=\dot{n}_{E_{e}}^{(\gamma \gamma)}-\frac{\partial}{\partial E}\left[\left(P_{\mathrm{IC}}+P_{\mathrm{syn}}+P_{\mathrm{ad}}\right) n_{E_{e}}^{e}\right]+\dot{n}_{E_{e}}^{\mathrm{inj}}$,

$\dot{n}_{E_{\gamma}}^{\gamma}=-\frac{n_{E_{\gamma}}^{\gamma}}{t_{\gamma \gamma}}-\frac{n_{E_{\gamma}}^{\gamma}}{t_{\mathrm{comp}}^{\mathrm{nb}}}-\frac{n_{E_{\gamma}}^{\gamma}}{t_{\mathrm{esc}}^{\mathrm{nb}}}+\dot{n}_{E_{\gamma}}^{\text {(IC) }}+\dot{n}_{E_{\gamma}}^{\text {(syn) }}$,

where $t_{\gamma \gamma}$ is the two-photon annihilation time, $t_{\text {comp }}^{\mathrm{nb}}$ is the energyloss time due to Compton scatterings in the nebula, $t_{\mathrm{esc}}^{\mathrm{nb}}=R_{\mathrm{nb}} / \mathrm{c}$ is 
the photon escape time for non-thermal photons, and $P_{\mathrm{IC}}, P_{\mathrm{syn}}$, and $P_{\text {ad }}$ are energy-loss rates due to the IC, synchrotron radiation and adiabatic expansion, respectively. Note that different from Murase et al. $(2015,2016)$, we take into account both electromagnetic cascades and contributions from relic pairs that are injected at $t<$ $t_{\mathrm{sd}}$. These pairs are relevant at $t>t_{\mathrm{sd}}$ if $q_{1} \lesssim 1$. The lepton injection rate $\dot{n}_{E_{e}}^{\text {inj }}$ is determined by

$E_{e}^{2} \dot{n}_{E_{e}}^{\text {inj }}=\frac{3 \epsilon_{e} L_{\mathrm{sd}}}{4 \pi R_{\mathrm{nb}}^{2} c \mathcal{R}_{0}}\left\{\begin{array}{l}\left(\gamma_{e} / \gamma_{b}\right)^{2-q_{1}}\left(\varepsilon \leq \varepsilon_{b}\right) \\ \left(\gamma_{e} / \gamma_{b}\right)^{2-q_{2}}\left(\varepsilon_{b}<\varepsilon\right)\end{array}\right.$,

where $q_{1}<2$ and $q_{2} \geq 2$ are injection spectral indices. The observations of known Galactic PWNe suggest that a significant fraction of the spin-down power is dissipated inside or around the termination shock (e.g. Tanaka \& Takahara 2013), and we take $\epsilon_{e}=$ $1-\epsilon_{B}$. Note that efficient conversion from the rotation energy to the particle energy is also necessary to explain the observed optical emission in the pulsar/magnetar-driven scenario. We treat $\gamma_{b}$ as a parameter, assuming $\gamma_{e} \in\left[10^{3}, 10^{7}\right]$, where $\gamma_{b}$ does not have to be the same as the bulk Lorentz factor of the wind. The pair multiplicity is model dependent, and cascades in the nebula and/or wind may contribute at early times (Murase et al. 2015; Vurm \& Metzger 2021). Also, it has been known that the radio data of the Crab nebula require large multiplicities (with $\sim 10^{6}$ ) that are theoretically challenging (e.g. Arons 2012), which may originate from continuously heated pairs that were injected in the past or from another component (Atoyan 1999; Tanaka \& Asano 2017). Thus, we use Equation (14) to allow such possibilities, in which the effective pair multiplicity can be expressed by $\gamma_{b}, q_{1}$ and $q_{2}$ (Murase et al. 2015). Leptons above $\gamma_{b}$ may be accelerated in shocks, but will not directly affect the radio flux. We adopt $q_{2}=2.5$ throughout this work, and note that this choice does not significantly affect our results on radio fluxes.

With respect to absorption processes, we implement free-free absorption and the Razin-Tsytovich suppression in the ejecta as well as SSA in the nebula. Following Murase et al. (2016), we consider two representative cases with and without absorption in the singly ionized CO ejecta. In reality, clumpiness or asymmetry in the ejecta leads to low-density regions through which the radio emission can escape more easily (e.g. Suzuki \& Maeda 2021). Note that the recent detection of radio emission from PTF10hgi could be explained by quasi-steady emission from nascent PWNe, for which the required absorption coefficient lies within the two limits (Eftekhari et al. 2019, 2020; Law et al. 2019; Hatsukade et al. 2021).

\section{IMPLICATIONS OF HIGH-FREQUENCY RADIO DATA}

\subsection{ALMA and NOEMA observations}

Working from the Open Supernovae Catalogue (Guillochon et al. 2017), Omand et al. (2018) investigated the detectability of radio and millimetre emission from the recent brightest six SLSNe with goodquality data of SN light curves. We further supplemented those events with four SLSNe that occurred over 2015-16. Spin-down parameters, $B_{*}$ and $P_{i}$, were estimated for each . Among these 10 SLSNe, we found just two objects, SN 2015bn and SN 2016ard, to be promising mm targets for ALMA. Subsequently, the nearby SN 2017egm was discovered and added to our sample, with observations by ALMA and NOEMA conducted. See Table 1 for the model parameters of SN 2015bn, SN 2016ard, and SN 2017egm. The ALMA data were analysed with the CASA (Common Astronomy Software Application) software package (McMullin et al. 2007). The data were taken in the
Time Division Mode at ALMA Bands 3 and 6. The bandwidths were $7.5 \mathrm{GHz}$, and two polarization products $\mathrm{XX}$ and $\mathrm{YY}$ were obtained to produce the Stokes I image. The NOEMA data were analysed with the GILDAS (Grenoble Image and Line Data Analysis Software) ${ }^{1}$ package. The data were taken in dual-polarization at NOEMA band 1 , which includes two side bands centred at 86.26 and $101.74 \mathrm{GHz}$ with $16 \mathrm{GHz}$ bandwidth each, giving the total coverage of $32 \mathrm{GHz}$. With standard imaging techniques used in CASA and GILDAS, we obtain flux densities and root-mean-square values in the image within 5 arcsec, and $3 \sigma$ upper limits are reported in Table 2 . We have confirmed that the sensitivity levels achieved by these observations are consistent with expectations, given the noise and integration times.

\subsubsection{SN 2015bn}

SN 2015bn is among the best studied SLSNe-I (e.g. Nicholl et al. 2016a,b). It was discovered by the Catalina Sky Survey on 2014 December 23, and later observed by the Mount Lemmon Survey and the Pan-STARRS Survey. The sky coordinates are RA $=11^{\mathrm{h}} 33^{\mathrm{m}} 41^{\mathrm{s}}$ and $\delta=+00^{\circ} 43^{\prime} 32^{\prime \prime}$ (J2000.0), and the measured redshift is $z=$ 0.1136 . The optical light curve reached its peak on MJD 57102, which is $116 \mathrm{~d}$ after the explosion time $T_{i}$ in our model. We adopt the values of $B_{*}, P_{i}$, and $M_{\mathrm{ej}}$ obtained by Omand et al. (2018) with the $V$ band data. ALMA observations at Band $3(\sim 3 \mathrm{~mm})$ and Band $6(\sim 1.3 \mathrm{~mm})$ were conducted on 2017 April 10 and 8, respectively, about $1100 \mathrm{~d}$ after the peak time $T_{\mathrm{pk}}$. The numbers of antennas were 46 and 43, respectively, for which 1 Execution Block (EB) was taken. The beam sizes were $1.87 \mathrm{arcsec}$ by $1.55 \mathrm{arcsec}$ at Band 3 and 1.02 arcsec by 0.64 arcsec at Band 6, respectively. The data were calibrated and imaged by the ALMA Pipeline version ver40896 with the CASA version 5.1.1-5. No significant emission was found. Note that these observations are independent of those reported by Eftekhari et al. (2020) at $100 \mathrm{GHz}$.

\subsubsection{SN 2016ard}

SN2016ard was found by Pan-STARRS1 on 2018 February 14 (Chornock et al. 2016). Its sky coordinates are $\mathrm{RA}=14^{\mathrm{h}} 10^{\mathrm{m}} 44^{\mathrm{s}}$ and $\delta=-10^{\circ} 09^{\prime} 35^{\prime \prime}(\mathrm{J} 2000.0)$, and its redshift is $z=0.2025$. The SN light curve in the $w$ band reached the maximum on MJD 57463, which is $74 \mathrm{~d}$ after the explosion in our model. ALMA data at Bands 3 and 6 were taken on 2018 July 18-19 and June 23-28, respectively, about $900 \mathrm{~d}$ after $T_{\mathrm{pk}}$. Correspondingly, 2 and 6 EBs were obtained with 45 antennas and 45-47 antennas, respectively. The beam sizes were $3.17 \operatorname{arcsec}$ by $2.23 \operatorname{arcsec}$ at Band 3 and 1.27 arcsec by $0.94 \operatorname{arcsec}$ at Band 6, respectively. For each band, we used the ALMA Pipeline version ver40896 with the CASA version 5.1.1-5 and the ALMA Pipeline version ver42030M with the CASA version 5.4.0-68. Although the $100 \mathrm{GHz}$ image had a $\sim 1 \sigma$ fluctuation, no significant emission was found.

\subsubsection{SN 2017egm}

SN 2017egm was discovered by the Gaia satellite on 2017 May 23 (Bose et al. 2018). Its sky coordinates are $\mathrm{RA}=10^{\mathrm{h}} 19^{\mathrm{m}} 05^{\mathrm{s}}$ and $\delta=$ $+46^{\circ} 27^{\prime} 14^{\prime \prime}$ (J2000.0), and its redshift is $z=0.03072$. This SLSN-I, associated with the massive spiral galaxy NGC 3231, is one of the nearest SLSNe. The SN light curve reached its optical maximum on MJD 57463, which is 51 days after the explosion in our model. The

\footnotetext{
${ }^{1}$ http://www.iram.fr/IRAMFR/GILDAS
} 
Table 1. Model parameters used for fitting optical light curves of three SLSNe. Initial periods are investigated from $1.0 \mathrm{~ms}$ to $P_{\max }$. For $P_{i}=1 \mathrm{~ms}$, the high magnetization model is also considered (see text for details), for which the parameters are indicated in parentheses.

\begin{tabular}{lccccccc}
\hline SN name & $z$ & $T_{\mathrm{pk}}[\mathrm{MJD}]$ & $B_{*, 13}$ at $P=1 \mathrm{~ms}$ & $M_{\mathrm{ej}}\left(\mathrm{M}_{\odot}\right)$ at $P=1 \mathrm{~ms}$ & $P_{\max }(\mathrm{ms})$ & $B_{*, 13}$ at $P_{\max }$ & $M_{\mathrm{ej}}\left(\mathrm{M}_{\odot}\right)$ at $P_{\max }$ \\
\hline 2015bn & 0.1136 & 57102 & $2.1(1.0)$ & $17(8.5)$ & 1.4 & 1.0 & 5.0 \\
2016ard & 0.2025 & 57463 & $6.0(3.0)$ & $12(6.0)$ & 2.2 & 1.7 & 1.5 \\
2017egm & 0.03072 & 57922 & $13(6.5)$ & $11.5(5.7)$ & 2.0 & 2.0 & 2.0 \\
\hline
\end{tabular}

Table 2. Summary of ALMA (upper columns) and NOEMA (lower columns) observations of the three SLSNe. The former was based on the project codes 2017.1.00975.S (PI: K. Murase) and 2018.1.01295.S (PI: D. Coppejans), and the latter was based on the project ID S18BH (PI: C. Omand). Note that $T=$ $t+T_{i}$.

\begin{tabular}{lcccc}
\hline SN name & $\begin{array}{c}T \\
(\mathrm{MJD})\end{array}$ & $\begin{array}{c}\text { Integration time } \\
(\mathrm{s})\end{array}$ & $\begin{array}{c}\text { Frequency } \\
(\mathrm{Hz})\end{array}$ & $\begin{array}{c}\text { Upper limit } \\
(\mu \mathrm{Jy})(3 \sigma)\end{array}$ \\
\hline 2015bn & 58218 & 333 & 97.1 & 87.4 \\
& 58216 & 666 & 233.0 & 123 \\
2016ard & $58317-58318$ & 5746 & 97.5 & 26.0 \\
& $58292-58297$ & 28123 & 233.0 & 15.1 \\
2017egm & 58591 & 1182 & 97.5 & 134 \\
& 58587 & 2331 & 233.0 & 151 \\
2017egm & 58342 & 6800 & $86.3 / 101.7$ & \\
& 58349 & 9400 & $86.3 / 101.7$ & \\
& 58368 & 9400 & $86.3 / 101.7$ & \\
& 58381 & 4000 & $86.3 / 101.7$ & \\
& 58382 & 4000 & $86.3 / 101.7$ & \\
& 58385 & 6800 & $86.3 / 101.7$ & \\
& $58342-58385$ & 40300 & 86.3 & 40.4 \\
& $(58365: \mathrm{avg})$ & 40300 & 101.7 & 43.5 \\
\hline
\end{tabular}

parameters, $B_{*}, P_{i}$, and $M_{\mathrm{ej}}$, are determined using the $V$ band data. NOEMA data were taken during six epochs between 2018 August 12 and September 24 and calibrated and imaged using GILDAS version nov18a to obtain time-integrated, polarization-averaged upper limits at 86 and $102 \mathrm{GHz}$. The corresponding beam sizes were 4.99 arcsec by $4.92 \operatorname{arcsec}$ and 4.41 arcsec by 4.30 arcsec, respectively. Emission was detected from the host galaxy at an offset of 13 arcsec from the SN position, consistent with the location of a known star-forming region (Nicholl et al. 2017). ALMA observations at Bands 3 and 6 were conducted on 2019 April 18-14, respectively. The numbers of antennas were 48 and 43, respectively, for which 1 EB was taken. The data were calibrated and imaged by the ALMA Pipeline version ver42254M with the CASA version 5.4.0-70. The beam sizes were $2.69 \operatorname{arcsec}$ by $0.92 \operatorname{arcsec}$ at Band 3 and 1.17 arcsec by 0.35 arcsec at Band 6, respectively.

\subsection{Model implications}

Using the method described in Section 2.2, we calculate non-thermal PWN spectra in a time-dependent manner. The evolution of nascent PWNe is mainly governed by $B_{*}, P_{i}$, and $M_{\mathrm{ej}}$, which are determined through modeling SN optical light curves. We fit the SN data by eye as in Omand et al. (2018) and Law et al. (2019), which can determine parameters to within 5-10 per cent. The optical opacity, nickel mass, and initial ejecta energy are fixed to $K=0.1 \mathrm{~cm}^{2} \mathrm{~g}^{-1}, M_{\mathrm{Ni}}=0.1 \mathrm{M}_{\odot}$, and $\mathcal{E}_{\text {ej }}=10^{51} \mathrm{erg}$, respectively. In SLSNe, these parameters are less relevant than the above three main parameters listed in Table 1. For non-thermal emission in the radio and millimetre bands, three microphysical parameters $\left(\epsilon_{B}, \gamma_{b}, q_{1}\right)$ are also relevant, for which we examine the following three models.

\subsubsection{Crab-motivated model}

As the fiducial assumption, following Murase et al. (2015), we postulate that the magnetization and lepton injection spectrum are similar to those inferred from young Galactic PWNe. In particular, motivated by the Crab nebula, we take $\epsilon_{B}=0.003, \gamma_{b}=6 \times 10^{5}$, and $q_{1}=1.5$ (Tanaka \& Takahara 2010). Note that $q_{1}>1$ is obtained by other studies (e.g. Atoyan \& Aharonian 1996) and favoured by modeling of radio emission from PTF 10hgi (Law et al. 2019). Results are shown in Figs 2 and 3. Because electrons and positrons are in the fast cooling regime, i.e. $\gamma_{c}<\gamma_{b}$, the resulting synchrotron spectra should be softer than those of Galactic PWNe. For SN2015bn and SN 2017egm, light curves at 100 and $230 \mathrm{GHz}$ have peaks at $\sim 600-1000 \mathrm{~d}$, depending on details of the free-free absorption in the ejecta. With $P_{i}=1 \mathrm{~ms}$, the flux predictions for SN 2016ard and SN 2017egm are allowed by the data, while the models with $P_{i}=P_{\max }$ are ruled out. On the other hand, we find that the model fluxes of $\mathrm{SN}$ 2015 bn and SN 2017egm have $\sim(2-3) \sigma$ tensions with the nondetections at $230 \mathrm{GHz}$ even for $P_{i}=1 \mathrm{~ms}$. In this case, using $F_{v} \lesssim 100 \mu \mathrm{Jy}$ at this frequency (see Table 2), we have the following constraint

$\gamma_{b, 5.8}^{-1 / 2} \epsilon_{B,-2.5}^{-1 / 8}\left(1+Y_{\text {IC }}\right)^{-1} \lesssim 1$.

One sees that this requirement can be relaxed by increasing $\gamma_{b}$ and/or $\epsilon_{B}$ and/or $Y_{\text {IC }}$, and we consider two alternative models.

\subsubsection{Low-magnetization model}

One of the solutions to reduce the radio flux is to increase $Y_{\text {IC }}$. Murase et al. (2015) showed that external inverse-Compton emission is important until $\mathrm{SN}$ photons mostly escape. Intriguingly, extremely small values of $\epsilon_{B}$ are independently motivated by a possible solution to the missing energy problem for SN 2015bn and SN 2017egm (Vurm \& Metzger 2021). As an example, we take $\epsilon_{B}=10^{-6}, \gamma_{b}=10^{3}$, and $q_{1}=1$ (in which the Compton parameter $Y_{\mathrm{IC}} \gtrsim 10$ ). Results are shown in Figs 2 and 3, where we see that the synchrotron fluxes can be lower by an order of magnitude. Note that the spectrum is softer because the synchrotron peak is lower.

\subsubsection{High-magnetization model}

Here, we consider an alternative model to satisfy the ALMA and NOEMA constraints, in which we adopt $\epsilon_{B}=0.5, \gamma_{b}=10^{7}$, and $q_{1}=1$. The magnetization around the termination shock may be as large as $\gtrsim 1$. Although too large values cannot explain optical SN emission, dissipation and thermalization of the Poynting energy in the pulsar wind may be more inefficient. Such high-magnetization models inevitably give a stronger magnetic field in the nebula, $B_{\mathrm{nb}} \simeq 24 \mathrm{G} \epsilon_{B,-0.3}^{1 / 2} P_{i,-3}^{1 / 2} M_{\mathrm{ej}, 1}^{3 / 4} t_{\mathrm{yr}}^{-3 / 2}$, and the synchrotron frequency, $v_{b} \simeq 1.0 \times 10^{22} \mathrm{~Hz} \gamma_{b, 7}^{2} \epsilon_{B,-0.3}^{1 / 2} P_{i,-3}^{1 / 2} M_{\mathrm{ej}, 1}^{3 / 4} t_{\mathrm{yr}}^{-3 / 2}$, is expected in the $\mathrm{MeV}$ range. Note that the fast cooling nebular spectrum is flatter than that observed in the Crab nebula (Murase et al. 2016). In this 

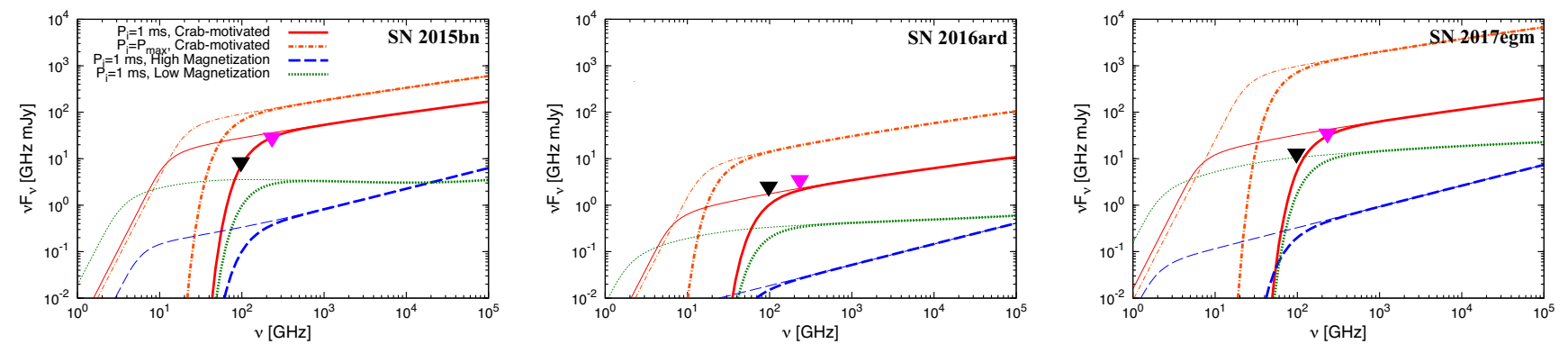

Figure 2. Non-thermal spectra of SN 2015bn (left-hand panel), SN 2016ard (middle panel), and SN 2017egm (right-hand panel), at ALMA observation times for Band 3. Thick/dashed curves indicate synchrotron fluxes with/without ejecta absorption.
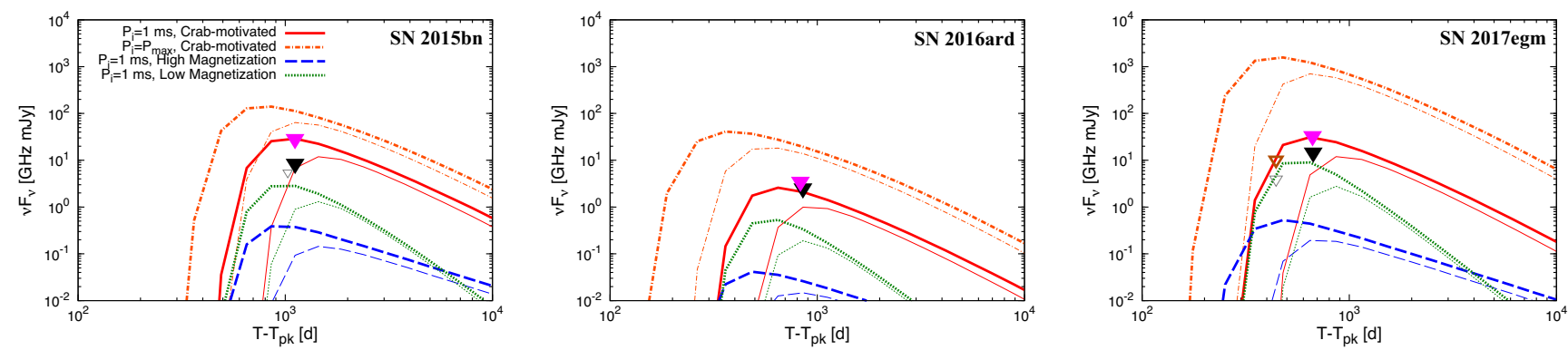

Figure 3. High-frequency radio light curves of SN 2015bn (left-hand panel), SN 2016ard (middle panel), and SN 2017egm (right-hand panel) at two frequency bands. Thick/thin curves represent model light curves at the 230/100 GHz band. Correspondingly, ALMA upper limits at Bands 3 and 6 are shown as filled black and pink triangles, respectively. In the right-hand panel, thick-brown/thin-grey open triangles indicate NOEMA upper limits at the 100/90 GHz band. The grey open triangle in the left-hand panel indicates the upper limit reported by Eftekhari et al. (2020).

model, we have $F_{v} \simeq 5.7 \mu \mathrm{Jy} \gamma_{b, 7}^{-1} \epsilon_{B,-0.3}^{-1 / 4} v_{11}^{-1 / 2} B_{*, 13}^{-2} P_{i,-3}^{-1 / 4} M_{\mathrm{ej}, 1}^{-3 / 8} t_{\mathrm{yr}}^{-5 / 4}$ $\left[\mathcal{R}_{0}\left(1+Y_{\mathrm{IC}}\right)\right]^{-1}(d / 0.5 \mathrm{Gpc})^{-2}$ and $v_{\mathrm{sa}} \sim 4.3 \mathrm{GHz} \gamma_{b, 7}^{-1 / 3} B_{*, 13}^{-2 / 3} P_{i,-3}^{2 / 3}$ $M_{\mathrm{ej}, 1}^{1 / 3} t_{\mathrm{yr}}^{-4 / 3}\left[\mathcal{R}_{0}\left(1+Y_{\mathrm{IC}}\right)\right]^{-1 / 3}$, consistent with our numerical results shown in Figs 2 and 3. We also confirmed that thermal and nonthermal emission in this model is consistent with the late-time observations at the optical and X-ray bands, respectively (Bhirombhakdi et al. 2018).

\subsection{Radio-gamma-ray connection}

ALMA and NOEMA observations imply that the magnetization parameter may be significantly different from that observed in the Crab nebula. Here, we point out that high- and low-magnetization scenarios can be tested not only by high-frequency radio observations but also by soft $\gamma$-ray observations. As argued in Murase, Thompson \& Ofek (2014), sub-GeV $\gamma$-rays can escape as early as optical photons. The Bethe-Heitler process is relevant for sub-GeV gamma rays, and its optical depth is $\tau_{\mathrm{BH}} \approx\left(8 \sigma_{\mathrm{BH}} / \sigma_{T}\right) \tau_{T} \sim 10 \alpha_{\mathrm{em}} \tau_{T}$ for the CO ejecta, where $\tau_{T}$ is the Thomson optical depth and $\alpha_{\mathrm{em}} \simeq 1 / 137$. Then, the gamma-ray breakout time is (Murase et al. 2015)

$t_{\gamma-\mathrm{bo}}=t_{\mathrm{pk}} \sqrt{\frac{K_{\gamma}}{K \beta_{\mathrm{ej}}}} \sim t_{\mathrm{pk}} K_{\gamma,-2}^{1 / 2} K_{-1}^{-1 / 2} \beta_{\mathrm{ej},-1}^{-1 / 2}$,

where $K_{\gamma} \sim 0.01 \mathrm{~g}^{-1} \mathrm{~cm}^{2}$ is the opacity at $0.1 \mathrm{GeV}$.

In the high magnetization model, the peak energy of the intrinsic nebular spectrum, $E_{b}=h v_{b}$, is close to the synchrotron cutoff at $E_{M} \equiv h v_{M} \sim 160 \mathrm{MeV}$, which may be detected by gamma-

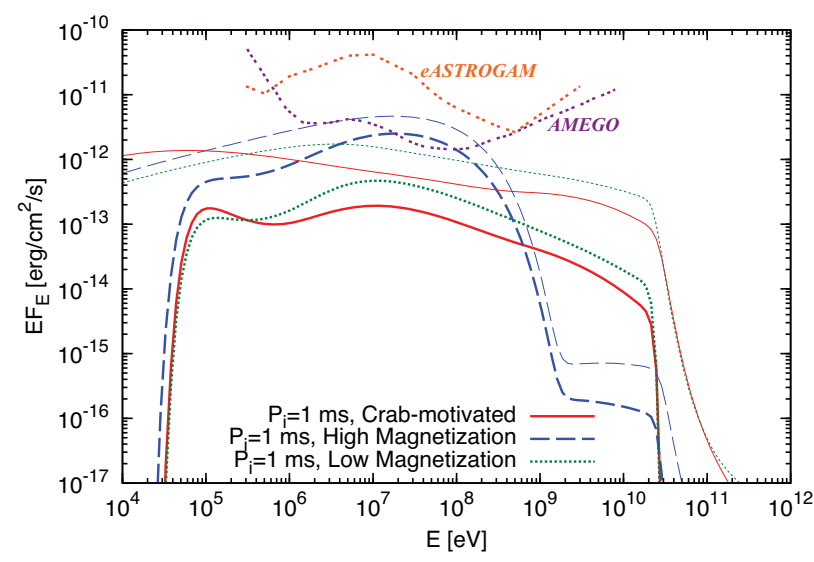

Figure 4. Gamma-ray fluxes from SN 2017egm at $T_{\mathrm{pk}}$. Cases with/without gamma-ray attenuation in the SN ejecta are shown by thick (thin) curves. Sensitivities of eASTROGAM and AMEGO are shown for an integration time of $10^{6} \mathrm{~s}$.

ray telescopes sensitive to sub-GeV $\gamma$-rays. As an example, the result for SN 2017egm is shown in Fig. 4. The expected gammaray signal is difficult to detect with Fermi-LAT with its sensitivity of $\sim 10^{-11} \mathrm{erg} \mathrm{cm}^{-2} \mathrm{~s}^{-1}$, but future $\mathrm{MeV} \gamma$-ray telescopes such as AMEGO and eASTROGAM will have the sensitivity to probe the predicted fluxes around the $\gamma$-ray breakout time. The predictions are different from the lower magnetization models, in which the 
inverse-Compton mechanism leads to gamma-rays beyond $\mathrm{GeV}$ energies (Murase et al. 2015, 2018).

The high-magnetization model also has a simple prediction for the relationship between radio and $\gamma$-ray fluxes

$$
\frac{\left.\nu F_{\nu}\right|_{\text {soft } \gamma}}{\left.v F_{v}\right|_{\text {radio }}} \approx\left(2.42 \times 10^{9}\right)^{2-\beta}\left(\frac{E_{\gamma}}{1 \mathrm{MeV}}\right)^{2-\beta}\left(\frac{v}{100 \mathrm{GHz}}\right)^{\beta-2},
$$

which leads to $\left(\left.v F_{v}\right|_{\text {soft } \gamma}\right) /\left(\left.v F_{v}\right|_{\text {radio }}\right) \sim 5 \times 10^{4}$ for $\beta=1 / 2$ and $E_{\gamma}=$ $h v<h v_{b}$. This is in contrast to the other two scenarios, in which the $\mathrm{GeV} \gamma$-rays are more prominent. Note that these results may be modified by attenuation in the ejecta (see Fig. 4).

\section{SUMMARY AND DISCUSSION}

The pulsar/magnetar-driven scenario for SLSNe-I naturally predicts synchrotron emission from embryonic PWNe. Here, we have reported ALMA and NOEMA observations of three SLSNe, SN 2015bn, SN 2016ard, and SN 2017egm, at ages of 1-3 yr, which set new upper limits on their high-frequency radio emissions. We also presented details of a theoretical model to calculate nebular emission that can be approximated by the analytical prescription. In particular, for SN 2015bn, we found that the upper limit at Band 6 $(\sim 230 \mathrm{GHz})$ disfavours the model if the nebular magnetization and lepton spectrum are similar to those for the Crab nebula. On the other hand, for SN 2016ard and SN 2017egm, the Crab-motivated model is still consistent with the ALMA and NOEMA limits, respectively, unless $P \sim P_{\max }$.

The present millimetre limits (see also Law et al. 2019; Eftekhari et al. 2020) are not sufficient to cover the relevant parameter space allowed by optical data, so further observations at the $\mathrm{mm}$ band are necessary to critically test the pulsar/magnetar-driven scenario. Dust emission observed at higher frequencies also enable us to probe the pulsar/magnetar central engine (Omand, Kashiyama \& Murase 2019). Nevertheless, our results demonstrate that high-frequency radio emission provides a powerful probe for non-thermal activity associated with young SLSNe. We investigated alternative high- and low-magnetization models. In either case, the predicted synchrotron flux is well below the current upper limits, while avoiding the missing energy problem in SN 2015bn (Bhirombhakdi et al. 2018). In particular, the high magnetization model leads to prominent synchrotron emission in the $1-100 \mathrm{MeV}$ range, which are good targets for future $\gamma$-ray observatories such as AMEGO (Moiseev et al. 2018) and eASTROGAM (De Angelis et al. 2017).

We note that high-frequency radio emission can also be produced in other scenarios for SLSNe. SLSNe may be accompanied by jets and the resulting off-axis jet emission may produce variable radio emission (Eftekhari et al. 2020; Hatsukade et al. 2021). An alternative mechanism is the interaction-powered scenario, in which particles are accelerated at the shocks between the SN ejecta and dense circumstellar material (Sorokina et al. 2016). Synchrotron signatures of secondary electrons and positrons produced via $p p$ interactions are expected (Murase et al. 2014). Such a late interaction has been seen for Type Ibc SNe, and it is promising even for SLSNe-I.

Fast-cooling nebular emission has been of interest as a counterpart signal of FRB sources and progenitors. The nascent nebular spectrum is predicted to have a steep spectrum with $\beta \geq 1.5$ (Murase et al. 2016) and $\left.v F_{v}\right|_{\text {radio }} /\left(\left.v F_{v}\right|_{\mathrm{X} \text {-ray }}\right) \gtrsim 8 \times 10^{-3}$. On the other hand, studies on Galactic PWNe infer $\beta \lesssim 1.4$ (Gaensler \& Slane 2006), consistent with the slow cooling spectrum. It is important to pursue a flexible approach that does not assume a Crab-like spectrum in interpreting non-detections of optical and X-ray counterparts. The quasi-steady synchrotron flux is sensitive to not only $B_{*}$ and $P_{i}$ but also the age $t=$
$T-T_{i}$. Slowly rotating magnetars, which are likely more common, are expected to yield lower radio fluxes. Thus, our model anticipates detectable persistent radio counterparts only for a fraction of FRBs like FRB 121102.

\section{ACKNOWLEDGEMENTS}

The ALMA observations were performed based on the Cycle 5 ALMA proposal with the project code 2017.1.00975.S, titled with 'Searching for the Smoking Gun of Magnetar-Powered SuperLuminous Supernovae' (PI: Kohta Murase) and the Cycle 6 ALMA proposal with the project code 2018.1.01295.S, titled with 'A Direct Test of the Magnetar-Model in SLSNe' (PI: Deanne Coppejans). ALMA is a partnership of ESO (representing its member states), NSF (USA) and NINS (Japan), together with NRC (Canada), MOST and ASIAA (Taiwan), and KASI (Republic of Korea), in cooperation with the Republic of Chile. The Joint ALMA Observatory is operated by ESO, AUI/NRAO, and NAOJ. The National Radio Astronomy Observatory is a facility of the National Science Foundation operated under cooperative agreement by Associated Universities, Inc. The NOEMA observations were performed based on the Summer 2018 NOEMA proposal with the project ID S18BH, titled 'Testing the Magnetar-Powered Scenario for Super-Luminous Supernovae with NOEMA' (PI: Conor Omand). The work of KM is supported by the Alfred P. Sloan Foundation, NSF Grant nos AST-1908689, AST-2108466, AST-2108467, and KAKENHI nos 20H01901 and 20H05852. CMBO has been supported by the Grant-in-aid for the Japan Society for the Promotion of Science (18J21778). The authors would like to thank Michel Bremer and Jan Martin Winters for their help with NOEMA data reduction.

\section{DATA AVAILABILITY}

The ALMA data presented in this paper are available at the ALMA archive using the project codes 2017.1.00975.S (PI: Kohta Murase) and 2018.1.01295.S (PI: Deanne Coppejans). The NOEMA data can be found the IRAM archive using the project ID S18BH001 (PI: Conor Omand). The theoretical model data underlying this article will be shared on reasonable request to the corresponding author.

\section{REFERENCES}

Arons J., 2012, Space Sci. Rev., 173, 341

Atoyan A. M., 1999, A\&A, 346, L49

Atoyan A. M., Aharonian F. A., 1996, MNRAS, 278, 525

Bhirombhakdi K., Chornock R., Margutti R., Nicholl M., Metzger B. D. Berger E., Margalit B., Milisavljevic D., 2018, ApJ, 868, L32

Bose S. et al., 2018, ApJ, 853, 57

Chatzopoulos E., Wheeler J. C., Vinko J., Horvath Z. L., Nagy A., 2013, ApJ, 773,76

Chen K.-J., 2021, Int. J. Mod. Phys., 30, 15

Chevalier R. A., Fransson C., 1992, ApJ, 395, 540

Chornock R., Bhirombhakdi K., Katebi R., Blanchard P., Nicholl M., Berger E., 2016, Astron. Telegram, 8790, 1

Coppejans D. L. et al., 2018, ApJ, 856, 56

Coppejans D. L. et al., 2021a, Astron. Telegram, 14393, 1

Coppejans D. L. et al., 2021b, Astron. Telegram, 14418, 1

De Angelis A. et al., 2017, Exp. Astron., 44, 25

Eftekhari T. et al., 2019, ApJ, 876, L10

Eftekhari T. et al., 2020, ApJ, 912, 23

Gaensler B. M., Slane P. O., 2006, ARA\&A, 44, 17

Gal-Yam A., 2019, ARA\&A, 57, 305

Greiner J. et al., 2015, Nature, 523, 189

Gruzinov A., 2005, Phys. Rev. Lett., 94, 021101 
Guillochon J., Parrent J., Kelley L. Z., Margutti R., 2017, ApJ, 835, 64

Hatsukade B. et al., 2021, ApJ, 911, 6

Hotokezaka K., Kashiyama K., Murase K., 2017, ApJ, 850, 18

Inserra C. et al., 2013, ApJ, 770, 128

Kasen D., Bildsten L., 2010, ApJ, 717, 245

Kashiyama K., Murase K., 2017, ApJ, 839, L3

Kashiyama K., Murase K., Bartos I., Kiuchi K., Margutti R., 2016, ApJ, 818, 94

Kennel C. F., Coroniti F. V., 1984, ApJ, 283, 710

Kotera K., Phinney E. S., Olinto A. V., 2013, MNRAS, 432, 3228

Law C. J. et al., 2019, ApJ, 886, 24

Margalit B., Metzger B. D., Berger E., Nicholl M., Eftekhari T., Margutti R., 2018, MNRAS, 481, 2407

Margalit B., Berger E., Metzger B. D., 2019, ApJ, 886, 110

Margutti R. et al., 2018, ApJ, 864, 45

Matthews D. et al., 2021, Astron. Telegram, 14580, 1

McMullin J. P., Waters B., Schiebel D., Young W., Golap K., 2007, in Shaw R. A., Hill F., Bell D. J., eds, ASP Conf. Ser. Vol. 376, Astronomical Data Analysis Software and Systems XVI. Astron. Soc. Pac., San Francisco, p.127

Metzger B. D., Vurm I., Hascoët R., Beloborodov A. M., 2014, MNRAS, 437, 703

Metzger B. D., Margalit B., Kasen D., Quataert E., 2015, MNRAS, 454, 3311

Moiseev A. et al., 2018, PoS, ICRC2017, 798

Moriya T. J., Sorokina E. I., Chevalier R. A., 2018, Space Sci. Rev., 214, 59

Murase K., Mészáros P., Zhang B., 2009, Phys. Rev. D, 79, 103001

Murase K., Thompson T. A., Ofek E. O., 2014, MNRAS, 440, 2528

Murase K., Kashiyama K., Kiuchi K., Bartos I., 2015, ApJ, 805, 82
Murase K., Kashiyama K., Mészáros P., 2016, MNRAS, 461, 1498

Murase K. et al., 2018, ApJ, 854, 60

Nicholl M. et al., 2013, Nature, 502, 346

Nicholl M. et al., 2016a, ApJ, 826, 39

Nicholl M. et al., 2016b, ApJ, 828, L18

Nicholl M., Berger E., Margutti R., Blanchard P. K., Guillochon J., Leja J., Chornock R., 2017, ApJ, 845, L8

Omand C. M. B., Kashiyama K., Murase K., 2018, MNRAS, 474, 573

Omand C. M. B., Kashiyama K., Murase K., 2019, MNRAS, 484, 5468

Ostriker J. P., Gunn J. E., 1971, ApJ, 164, L95

Petroff E., Hessels J. W. T., Lorimer D. R., 2019, A\&A Rev., 27, 4

Slane P., 2017, Pulsar Wind Nebulae, Springer-Verlag, Berlin, p. 2159

Sorokina E., Blinnikov S., Nomoto K., Quimby R., Tolstov A., 2016, ApJ, 829,17

Spitkovsky A., 2006, ApJ, 648, L51

Suzuki A., Maeda K., 2021, ApJ, 908, 217

Tanaka S. J., Asano K., 2017, ApJ, 841, 78

Tanaka S. J., Takahara F., 2010, ApJ, 715, 1248

Tanaka S. J., Takahara F., 2013, MNRAS, 429, 2945

Tendulkar S. P. et al., 2017, ApJ, 834, L7

Thompson T. A., Chang P., Quataert E., 2004, ApJ, 611, 380

Vurm I., Metzger B. D., 2021, ApJ, 917, 28

Woosley S. E., 2010, ApJ, 719, L204

Xiao D., Wang F., Dai Z., 2021, Sci. China Phys. Mechan. Astron., 64, 249501

Yamasaki S., Totani T., Kiuchi K., 2018, PASJ, 70, 39

Zhang B., 2014, ApJ, 780, L21

This paper has been typeset from a $\mathrm{T}_{\mathrm{E}} \mathrm{X} / \mathrm{LT} \mathrm{E} \mathrm{X}$ file prepared by the author. 